Exot i c quant um hol onomy and hi gher-or der except i onal poi nt s in quant um ki cked tops

\begin{tabular}{|l|l|}
\hline 著者 & Tanaka At ushi, K m Sang Wbok, Cheon Taksu \\
\hline $\begin{array}{l}\text { j our nal or } \\
\text { publ i cat i on t i t l e }\end{array}$ & Physi cal Revi ew E \\
\hline vol une & 89 \\
\hline number & 4 \\
\hline page $r$ ange & 042904 1- 042904- 8 \\
\hline year & 2014 O4 \\
\hline URL & ht t p: //hdl . handl e. net /10173/1456 \\
\hline
\end{tabular}




\title{
Exotic quantum holonomy and higher-order exceptional points in quantum kicked tops
}

\author{
Atushi Tanaka* \\ Department of Physics, Tokyo Metropolitan University, Hachioji, Tokyo 192-0397, Japan \\ Sang Wook Kim \\ Department of Physics Education, Pusan National University, Busan 609-735, South Korea \\ Taksu Cheon ${ }^{\dagger}$ \\ Laboratory of Physics, Kochi University of Technology, Tosa Yamada, Kochi 782-8502, Japan
}

(Received 7 February 2014; published 7 April 2014)

\begin{abstract}
The correspondence between exotic quantum holonomy, which occurs in families of Hermitian cycles, and exceptional points (EPs) for non-Hermitian quantum theory is examined in quantum kicked tops. Under a suitable condition, an explicit expression of the adiabatic parameter dependencies of quasienergies and stationary states, which exhibit anholonomies, is obtained. It is also shown that the quantum kicked tops with the complexified adiabatic parameter have a higher-order EP, which is broken into lower-order EPs with the application of small perturbations. The stability of exotic holonomy against such bifurcation is demonstrated.
\end{abstract}

DOI: 10.1103/PhysRevE.89.042904

PACS number(s): 05.45.Mt, 03.65.Vf, 03.67.-a

\section{INTRODUCTION}

Along adiabatic time evolution, the state of a closed quantum system stays within an eigenspace of the Hamiltonian, when the system is initially prepared to be in a stationary state [1]. It is nevertheless possible that an adiabatic cycle induces nontrivial change. The most famous example reported by Berry is the appearance of the geometric phase factor [2]. A non-Abelian version of the quantum phase holonomy was subsequently reported by Wilczek and Zee [3]. Later, it was shown that an adiabatic cycle can induce the interchanges of eigenenergies and eigenspaces [4]. Namely, under the presence of such an exotic quantum holonomy, the initial and final states of an adiabatic cycle belong to different eigenspaces. The exotic quantum holonomy has been studied both in one-body [5-10] and in many-body systems [11]. Applications of the exotic quantum holonomy to quantum state manipulation and adiabatic quantum computation [12] were also proposed [5,6,13].

Quantum maps describing periodically kicked systems [14] are useful to investigate the exotic quantum holonomy. An adiabatic cycle for a family of the quantum maps induces a permutation among quasienergies, which are determined by the eigenvalues of Floquet operators [15], instead of their eigenenergies [5]. We believe the experimental verification of the exotic quantum holonomy is now feasible considering the recent development of technology [16-19]. The exotic quantum holonomy in autonomous systems, in contrast to periodically driven systems, requires either the divergence of the eigenenergies [4] or the exact crossing of eigenenergies [8].

It was recently pointed out that the exotic quantum holonomy is closely associated with Kato's exceptional points (EPs) located at the complexified parameter space outside the adiabatic cycle [20]. An EP is a degeneracy of a non-Hermitian square matrix. Two eigenvalues and eigenvectors of the $2 \times 2$ non-Hermitian matrix are interchanged after parametric evolution along the cycle encircling the EP, which resembles

\footnotetext{
*http://researchmap.jp/tanaka-atushi/

${ }^{\dagger}$ http://researchmap.jp/T_Zen/
}

the holonomic behavior of the exotic quantum holonomy. However, the time evolution along the cycle in the adiabatic limit generally does not induce the "flip" of stationary states due to the presence of the the decay process, which is inherent to non-Hermitian systems [21-23]. On the other hand, if a Hermitian cycle can be smoothly shrunk to the non-Hermitian one so that the time spent in encircling the cycle is short enough, such an interchange of eigenspace can take place within adiabatic time evolution. An example is a family of quantum kicked spin- $\frac{1}{2}$ [24], where the interchange between two eigenspaces in the two-level system due to the exotic quantum holonomy corresponds to the EP that resides in the complexified parameter space of the quantum kicked spin.

As far as many interacting levels are concerned, the situation becomes very complicated since it is possible to find multiple degeneracies. However, it is much easier to deal with the case that the adiabatic cycle encircles several EPs associated with only two levels. It means that the cycle contains many EPs, but each EP is doubly degenerate. This has been recently studied in Refs. $[25,26]$ by using a $3 \times 3$ nonHermitian matrix. The exotic quantum holonomy associated with multiple EPs is also studied in the two-body Lieb-Liniger model [27]. However, the multiply degenerated EP has been rarely investigated (see Ref. [28] for triple EPs and Ref. [29] for higher-order EPs).

In this paper, we show that highly degenerate EPs can be systematically constructed by using the quantum kicked top with appropriate parameters chosen. The degeneracy of the EPs is given as $2 J+1$, where $J$ is the magnitude of angular momentum $J$ of the top. We show that the exotic quantum holonomy of the kicked quantum top is intimately related to the highly degenerate EP.

The plan of this paper is the following. In Sec. II, we introduce a quantum top under a rank-1 kick. We briefly explain the consequence of the existence theorem of the exotic quantum holonomy [6]. In Sec. III, we show the presence of the exotic quantum holonomy using an explicit expression of the solution of the eigenvalue problem, instead of the existence theorem. This is possible only when the parameter 
of the system satisfies a solvable condition. This condition implies the presence of EPs with higher order, as shown in Sec. IV. We show that the higher-order EP is fragile against perturbations in Sec. V. We also explain the correspondence between the exotic quantum holonomy and the remnants of broken higher-order EPs. A summary is found in Sec. VI.

\section{QUANTUM TOP UNDER A RANK-1 KICK}

We introduce a quantum top (or spin) under a rank-1 kick [30] in this section. We show the quasienergy and eigenspace anholonomies of this model with the help of a theorem that ensures the existence of the exotic quantum holonomy for quantum map under a rank-1 perturbation $[5,6]$.

Let $\hat{\boldsymbol{J}}$ denote the angular momentum of the top. In the absence of kick, we suppose that the top rotates around the $z$ axis with an angular frequency $\omega$. A rank-1 kick $\lambda|v\rangle\langle v|$ is applied periodically in time, where $\lambda$ is the strength of the kick and $|v\rangle$ is a normalized vector. The time is normalized by the period of the kick. The system is described by the Hamiltonian

$$
\hat{H}(t)=\omega \hat{J}_{z}+\lambda|v\rangle\langle v| \sum_{n=-\infty}^{\infty} \delta(t-n) .
$$

We set $\hbar=1$ throughout this paper. We assume that $|v\rangle$ belongs to $d$-dimensional eigenspace of $\hat{\boldsymbol{J}}^{2}=J(J+1)$, where $J$ is either an integer or half integer, and $d=2 J+1$.

The Hamiltonian (1) can be experimentally implemented by using nuclear magnetic moment $\hat{\boldsymbol{J}}$ under the influence of static magnetic field $\omega \hat{J}_{z}$ and the periodic kick $|v\rangle\langle v|$ composed by a polynomial of $\hat{J}_{y}[18,31]$. The polynomial depends on $J$. For example, the rank-1 terms for $J=\frac{1}{2}, 1$, and $\frac{3}{2}$ are

$$
\hat{J}_{y}+\frac{1}{2}, \quad \frac{1}{2}\left(\hat{J}_{y}+1\right) \hat{J}_{y}, \quad \frac{1}{6}\left(\hat{J}_{y}+\frac{3}{2}\right)\left(\hat{J}_{y}+\frac{1}{2}\right)\left(\hat{J}_{y}-\frac{1}{2}\right),
$$

respectively. These represent quadrupolar or higher-order multipole interactions of nuclear momentum. We may replace $\hat{J}_{y}$ with $\hat{\boldsymbol{J}} \cdot \boldsymbol{n}$ for the above examples as long as a normalized vector $\boldsymbol{n}$ is not parallel to the $z$ axis.

We examine how the stationary states of the kicked top evolve when the kick strength $\lambda$ adiabatically varies. Since the kicked top is a periodically driven system, the stationary states are the eigenvectors of the Floquet operator describing a unitary time evolution during a unit time interval:

$$
\hat{U}(\lambda) \equiv e^{-i \omega \hat{J}_{z}} e^{-i \lambda|v\rangle\langle v|}
$$

The real parameter $\lambda$ is geometrically equivalent to a circle because of the $2 \pi$ periodicity of $\hat{U}(\lambda)$ [5], which can be easily seen with the expansion

$$
\hat{U}(\lambda)=e^{-i \omega \hat{J}_{z}}\left[(1-|v\rangle\langle v|)+\Lambda^{-1}|v\rangle\langle v|\right]
$$

with

$$
\Lambda=e^{i \lambda}
$$

Here $\lambda$ runs from 0 to $2 \pi$ along a unit circle denoted by $C$. Let $\left|\varphi_{n}(\lambda)\right\rangle(n=0, \ldots, d-1)$ be an eigenvector of $\hat{U}(\lambda)$, i.e.,

$$
\hat{U}(\lambda)\left|\varphi_{n}(\lambda)\right\rangle=z_{n}(\lambda)\left|\varphi_{n}(\lambda)\right\rangle,
$$

where $z_{n}(\lambda)$ is the corresponding eigenvalue. Since $\hat{U}(\lambda)$ is unitary, $z_{n}(\lambda)$ lies in the unit circle of the complex plane. We introduce the quasienergy of $E_{n}(\lambda)$ so as to satisfy

$$
z_{n}(\lambda)=e^{-i E_{n}(\lambda)} \text {. }
$$

According to the theorem shown in Refs. [5,6], the adiabatic cycle $C$ induces quasienergy and eigenspace anholonomies when two conditions are satisfied: (1) $\exp \left(-i \omega \hat{J}_{z}\right)$ has no spectral degeneracy and (2) all eigenvectors of $\exp \left(-i \omega \hat{J}_{z}\right)$ are neither parallel nor orthogonal to $|v\rangle$. The first is equivalent to a nonresonant condition,

$$
\omega \notin\left\{\frac{2 \pi q}{p} \mid q \text { and } p \text { are integer and } 0<|p|<d\right\} .
$$

The first condition together with the assumption $\langle J, M \mid v\rangle \neq 0$ for all $M=-J, \ldots, J$ implies the second condition, where $|J, M\rangle$ is the standard basis of the angular momentum. A typical example of $|v\rangle$ is

$$
|v\rangle \equiv \frac{1}{\sqrt{d}} \sum_{M=-J}^{J}|J, M\rangle,
$$

which will be employed below.

We now explain the permutation of quasienergies induced by the adiabatic cycle $C$. We arrange the quasienergies $\left\{E_{n}(\lambda)\right\}_{n=0}^{d-1}$ in the following order:

$$
0 \leqslant E_{0}(\lambda)<E_{1}(\lambda)<\cdots<E_{d-1}(\lambda)<2 \pi
$$

at $\lambda=0$. The increment of $\lambda$ by $2 \pi$ results in

$$
E_{n}(\lambda+2 \pi)= \begin{cases}E_{n+1}(\lambda) & \text { for } n=0, \ldots, d-2 \\ E_{0}(\lambda)+2 \pi & \text { for } n=d-1 .\end{cases}
$$

Thus an adiabatic cycle $C$ increases the quantum number by unity (with modulo $d$ ). We emphasize that such a
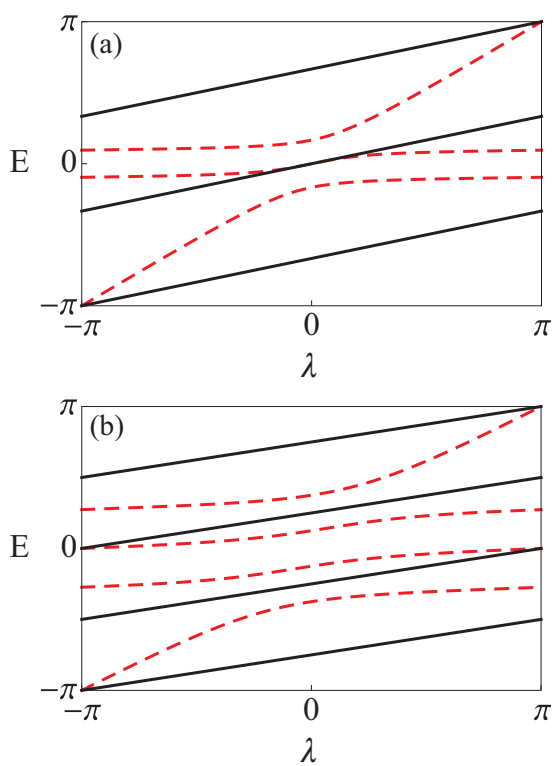

FIG. 1. (Color online) Parametric evolution of quasienergies of the quantum top under a rank-1 kick (3) along the unitary cycle $C$. (a) $J=1$ case. Thick and dashed curves correspond to $\omega=2 \pi / 3$ and $\pi / 6$, respectively. (b) $J=3 / 2$ case. Thick and dashed curves correspond to $\omega=\pi / 2$ and $\pi / 4$, respectively. As shown later in the main text, thick curves and dashed curves correspond to $(2 J+1)$-EP cases (Sec. IV) and 2EP cases (Sec. V), respectively. 
rearrangement of quasienergies occurs irrespective of $\omega$ and $|v\rangle$, as long as both the nonresonant condition (8) and the cyclic condition are satisfied. Several examples of the quasienergy anholonomy are shown in Fig. 1.

The quasienergy anholonomy directly implies the anholonomy in eigenspaces. Let us suppose that the state of the system is prepared in $|J, M\rangle$, which is a stationary state of the unperturbed Floquet operator $\hat{U}(\lambda)$ at $\lambda=0$. Equation (11) then implies that, after the completion of the adiabatic cycle along $C$, the state vector arrives at $|J, s(M ; C)\rangle$ up to a phase factor, where $s(M ; C)$ is the eigenvalue of $\hat{J}_{z}$ of the final state.

In order to determine $s(M ; C)$, we need to explicitly solve the eigenvalue problem of $\hat{U}(\lambda)$ at $\lambda=0$. It is straightforward to see that $|J, M\rangle(M=-J, \ldots, J)$ is an eigenvector corresponding to a quasienergy $\omega M$. Note that the mapping between the set of quasienergies $\{\omega M\}_{M=-J}^{J}$ and $\left\{E_{n}(\lambda)\right\}_{n=0}^{d-1}$, which satisfies Eq. (10), depends on $\omega$. We explore it with some specific examples of $J=\frac{1}{2}, 1$, and $\frac{3}{2}$ below.

First, we examine the case of $J=\frac{1}{2}$. For example, in the interval $0<\omega<2 \pi$ where the nonresonant condition (8) is always satisfied, we have

$$
E_{0}(0)=\frac{1}{2} \omega, \quad E_{1}(0)=2 \pi-\frac{1}{2} \omega,
$$

which correspond to $M=\frac{1}{2}$ and $-\frac{1}{2}$, respectively. According to Eq. (11), $E_{0}(\lambda)$ arrives at $E_{1}(0)$ at $\lambda=2 \pi$. Hence, the quantum state initially prepared to be in $|1 / 2,1 / 2\rangle$ at $\lambda=0$ is delivered to $|1 / 2,-1 / 2\rangle$ after the completion of the adiabatic cycle $C$. Similarly, the adiabatic cycle $C$ delivers $|1 / 2,-1 / 2\rangle$ to $|1 / 2,1 / 2\rangle$. This implies $s\left( \pm \frac{1}{2} ; C\right)=\mp \frac{1}{2}$.

Second, we examine the case $J=1$. We show that two intervals $0<\omega<\pi$ and $\pi<\omega<2 \pi$ provide different types of $s(M, C)$. Note that the nonresonant condition (8) is always satisfied in both intervals. In the former case,

$$
E_{0}(0)=0, \quad E_{1}(0)=\omega, \text { and } E_{2}(0)=2 \pi-\omega,
$$

which correspond to $M=0,1$, and -1 , respectively. Using a similar argument applied to the $J=\frac{1}{2}$ case mentioned above, we find $s(0 ; C)=1, s(1 ; C)=-1$, and $s(-1 ; C)=0$, which comprise a cyclic permutation. Namely, the itinerary of $|J, M\rangle$ induced by the adiabatic cycle $C$ is

$$
|J, 0\rangle \mapsto|J, 1\rangle \mapsto|J,-1\rangle .
$$

For another interval $\pi<\omega<2 \pi$, we have

$$
E_{0}(0)=0, \quad E_{1}(0)=2 \pi-\omega, \quad E_{2}(0)=\omega
$$

to satisfy Eq. (10). Hence the corresponding adiabatic itinerary of $|J, M\rangle$ is

$$
|J, 0\rangle \mapsto|J,-1\rangle \mapsto|J, 1\rangle .
$$

Hence the itinerary is suddenly changed around a critical point $\omega=\pi$. In this sense, we may choose the itinerary by varying $\omega$. Now it is straightforward to extend the present analysis to arbitrary $J$ and $\omega$.

\section{EXPLICIT EXPRESSIONS OF EIGENVALUES AND EIGENVECTORS}

So far we have explained the exotic quantum holonomy of the kicked tops (1) using the general theorem shown in
Refs. [5,6]. In this section, we explain the details of the anholonomies with the help of the explicit expressions of quasienergy and eigenvectors for an arbitrary $J$. This is possible when we choose a "solvable" value of $\omega$. This also helps us to examine the complexification of $\lambda$, as shown in the following sections.

We here examine the case $\omega=2 \pi / d$. Note that the following argument is also applicable to the case $\omega=2 \pi r / d$ with an integer $0 \leqslant r<d$. We assume that $|v\rangle$ satisfies Eq. (9). This allows us to introduce a mapping of the kicked top into a kicked particle in a periodic lattice (or, a kicked tight-binding model). We introduce basis vectors

$$
|m\rangle \equiv \frac{1}{\sqrt{d}} \sum_{M=-J}^{J} e^{i 2 \pi M m / d}|J, M\rangle,
$$

which describes a localized state at the " $m$ th site" for $0 \leqslant m<$ $d$. From the assumption $\omega=2 \pi / d$, the unperturbed Floquet operator $\hat{U}(0)$ is

$$
\hat{U}(0)=\sum_{m=0}^{d-1}|m-1\rangle\langle m|,
$$

which describes a nondispersive motion of a particle in a one-dimensional periodic lattice. Since we have chosen $|v\rangle=$ $|m=0\rangle$ [see Eq. (9)], we obtain the Floquet operator in the $|m\rangle$ representation:

$$
\hat{U}(\lambda)=\sum_{m=1}^{d-1}|m-1\rangle\left\langle m\left|+\Lambda^{-1}\right| d-1\right\rangle\langle 0| .
$$

This implies that an extra phase factor $\Lambda^{-1}$ is added along a "hopping" from the zeroth to the $(d-1)$ th site. Equation (19) is represented in the matrix representation with basis vectors $\{|m\rangle\}_{m=0}^{d-1}$ as follows:

$$
U(\lambda)=\left[\begin{array}{ccccc}
0 & 1 & & & \\
& 0 & 1 & & \\
& & \ddots & \ddots & \\
0 & & & \ddots & 1 \\
\Lambda^{-1} & 0 & & & 0
\end{array}\right] .
$$

It is straightforward to obtain the characteristic equation of $\hat{U}(\lambda)$

$$
\operatorname{det}\{z-U(\lambda)\}=z^{d}-\Lambda^{-1}=0,
$$

whose solution is

$$
z_{M}(\lambda)=e^{-i(2 \pi M+\lambda) / d},
$$

and the corresponding quasienergy is

$$
E_{M}(\lambda)=\frac{\lambda+2 \pi M}{d},
$$

with $M=-J, \ldots, J$. We also find the corresponding normalized eigenvector

$$
\left|\xi_{M}(\lambda)\right\rangle=\frac{1}{\sqrt{d}} \sum_{n=0}^{d-1} e^{-i n E_{M}(\lambda)}|n\rangle .
$$


In terms of basis $\left|\xi_{M}(\lambda)\right\rangle$, the eigenspace holonomy is regarded as an increment of quantum number $M$, i.e.,

$$
\left|\left\langle\xi_{M^{\prime}}(\lambda+2 \pi) \mid \xi_{M}(\lambda)\right\rangle\right|^{2}=\delta_{M^{\prime}, M+1} \bmod d .
$$

On the other hand, we need to identify which $|J, M\rangle$ is parallel to $\left|\xi_{M^{\prime}}(0)\right\rangle$ for a given $M^{\prime}$ to completely understand the eigenspace anholonomy. We show that $\left|\xi_{M}(0)\right\rangle$ is parallel to $\left|J, M^{\prime}\right\rangle$ only if $M-M^{\prime}=0 \bmod d$, i.e.,

$$
\left\langle J, M^{\prime} \mid \xi_{M}(0)\right\rangle=\delta_{M, M^{\prime}} \bmod d .
$$

We emphasize that this is applicable to any arbitrary $J$.

\section{HIGHER-ORDER EXCEPTIONAL POINTS BEHIND EXOTIC QUANTUM HOLONOMY}

In this section, we examine the EPs in the kicked quantum tops (1) under the specific choice of parameter $\omega=2 \pi / d$, which is examined in the previous section. We will show that the degree or the multiplicity of the EP is $d$, the highest possible value of the degree. We denote such an EP as $d \mathrm{EP}$ to distinguish it from conventional EPs whose order is 2.

So far, we have assumed that $\lambda$ is real or, equivalently, $|\Lambda|$ is unity [see Eq. (5)]. From now on, we complexify $\lambda$ to investigate EPs. This makes $\hat{U}(\lambda)$ a nonunitary operator, which may be regarded as an effective time evolution operator that describes conditional measurement processes [32,33]. The expression of the eigenvalues [Eq. (22)] remains intact regardless of the complexification of $\lambda$. On the other hand, because $\hat{U}(\lambda)$ is no longer unitary when $\lambda$ is not real, the left and the right eigenvectors of $\hat{U}(\lambda)$ become different so that the left eigenvector is separately introduced as $\left\langle\xi_{M}^{\mathrm{L}}(\lambda)\right|$ [34]. Both eigenvectors read

$$
\begin{aligned}
& \left|\xi_{M}(\lambda)\right\rangle=\frac{1}{\sqrt{d}} \sum_{m=0}^{d-1}\left\{z_{M}(\lambda)\right\}^{m}|m\rangle, \\
& \left\langle\xi_{M}^{\mathrm{L}}(\lambda)\right|=\frac{1}{\sqrt{d}} \sum_{m=0}^{d-1}\left\{z_{M}(\lambda)\right\}^{-m}\langle m|,
\end{aligned}
$$

which satisfy $\left\langle\xi_{M^{\prime}}^{\mathrm{L}}(\lambda) \mid \xi_{M}(\lambda)\right\rangle=\delta_{M^{\prime} M}$.

The spectral degeneracy of $\hat{U}(\lambda)$ occurs when $\operatorname{Im} \lambda=-\infty$, where all eigenvalues accumulate at $z=0$. This can be easily understood from the matrix representation $U(\lambda)$ [Eq. (20)], which converges to the $d \times d$ nilpotent Jordan block in the limit $\operatorname{Im} \lambda \rightarrow-\infty$ :

$$
U(\lambda) \rightarrow\left[\begin{array}{ccccc}
0 & 1 & & & \\
& 0 & 1 & & \\
& & \ddots & \ddots & \\
& & & \ddots & 1 \\
0 & & & & 0
\end{array}\right]
$$

Hence we conclude that $\Lambda=\infty$ is an EP of the order $d$. Also, we find that, from the characteristic equation [Eq. (21)], there is another $d \mathrm{EP}$ at $\Lambda=0$, where the eigenvalues of $\hat{U}(\lambda)$ accumulate at $z=\infty$.

An EP is the branch point of eigenvalues. We choose the line from the $d \mathrm{EP}$ at the origin to $-\infty$ in the $\Lambda$ plane as the

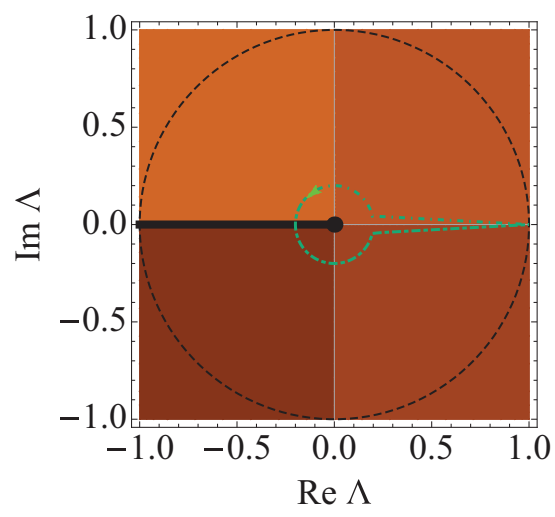

FIG. 2. (Color online) A contour plot of $\operatorname{Re} E_{-1}$ in the $\Lambda$ plane [ $\Lambda$ is defined in Eq. (5)]. Lighter (darker) color indicates large (smaller) value of $\operatorname{Re} E_{-1}$. Parameters are $J=1$ and $\omega=2 \pi /(2 J+1)$. There is a $3 \mathrm{EP}$ at $\Lambda=0$, which is indicated by the filled circle. A branch cut, which emanates from the $3 \mathrm{EP}$, is drawn by a bold line. A dash-dotted curve $C^{\prime}$ is a smooth deformation of $C$. The exotic quantum holonomy induced by $C$ can be emulated by the non-Hermitian cycle $C^{\prime}$.

branch cut represented by the thick horizontal line in Fig. 2. This choice is consistent with the analytic continuation of $z_{M}(\lambda)$ considered below. We start from the unit circle in the $\Lambda$ plane, where $\lambda$ is real valued. Because of the presence of the eigenvalue anholonomy, we need to introduce a discontinuous point of $z_{M}(\lambda)$ in the unit circle of $\Lambda$. Here we suppose $z_{M}(\pi+$ $0)=z_{M+1 \bmod 1}(\pi-0)$. Hence $z_{M}(\lambda)$ is discontinuous at $\Lambda=$ -1 in $C$. For each point in the unit circle of $\Lambda$, we extend $z_{M}(\lambda)$ along the radial direction in the $\Lambda$ plane. This uniquely specifies $z_{M}(\lambda)$ in the whole $\Lambda$ plane.

The variation of $\Lambda$ along $C$ (Fig. 2) induces permutation of the quasienergies,

$$
E_{M=-1} \mapsto E_{M=0} \mapsto E_{M=1} .
$$

This is an extension of the EP interpretation of the quasienergy anholonomy originally introduced in Ref. [24] to a family of multiple-level systems.

We explain an emulation of the exotic quantum holonomy with EPs by deforming the unitary cycle $C$ into non-Hermitian cycles, say, $C^{\prime}$. Suppose $C^{\prime}$ enclose the $d \mathrm{EP}$ and connect between the $d \mathrm{EP}$ and the initial point of the cycle $C$. This is depicted in Fig. 2, as for the $d=3$ case. Since the change of eigenvectors essentially occurs along the small cycle around the $d \mathrm{EP}$, we may say that only the contribution from the $d \mathrm{EP}$ is relevant.

\section{CORRESPONDENCE BETWEEN THE EXOTIC QUANTUM HOLONOMY AND THE FRAGMENTS OF $d$ EP}

Even a small perturbation can destroy a $d \operatorname{EP}(d>2)$. In this section, we examine the stability of the $d$ EPs of the quantum kicked top (3) at $\omega=2 \pi / d$, for $d=3$ and 4 . We show that the $d \mathrm{EP}$ is broken into 2EPs, the number of which is $d-1$, when we slightly vary $\omega$ from $2 \pi / d$. In contrast to such a catastrophe of the $d \mathrm{EPs}$, the exotic quantum holonomy is stable against such small perturbations. This raises another question of how the correspondence between the exotic quantum holonomy 


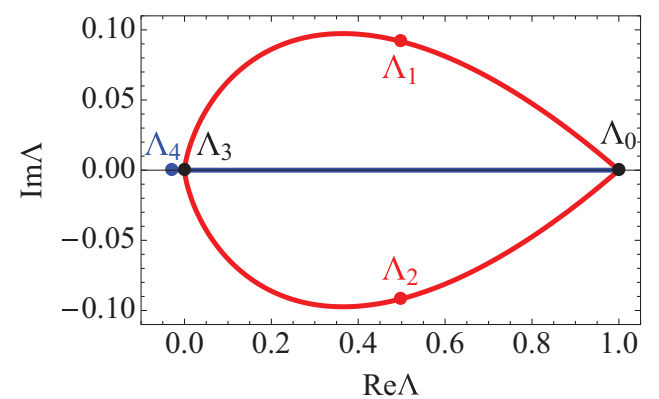

FIG. 3. (Color online) Parametric evolution of EPs of the quantum kicked top $J=1$ within the unitary cycle $C$ in the $\Lambda$ plane. $\omega$ is varied within the interval $[0, \pi]$. There is a triple degeneracy point $\Lambda_{0}(=1)$ at $\omega=0$. Note that the system is unitary (diagonalizable) at $\Lambda=\Lambda_{0}$. As $\omega$ is increased in the interval $(0,2 \pi / 3)$, two 2EPs evolve along the curves that pass $\Lambda_{1}$ and $\Lambda_{2}$ at $\omega=\pi / 6$. These 2EPs merge at $\omega=2 \pi / 3$ to form a $3 \mathrm{EP}$, which locates at $\Lambda_{3}(=0)$. If $\omega$ is increased further $(2 \pi / 3<\omega<\pi)$, there are two 2EPs, which are fragments of the 3EP $\Lambda_{3}$, in the real axis. Finally, at $\omega=\pi$, a 2EP arrives at $\Lambda_{0}$ to become a diabolic point, and the other arrives at $\Lambda_{4}(<0)$.

and the fragments of the $d \mathrm{EP}$ is established, which is also examined in this section.

We show a detailed analysis of the $J=1$ (i.e., $d=3$ ) case. In Fig. 3, we depict numerically obtained $\omega$ dependence of EPs. At $\omega=0$, we have a triple degeneracy point at $\Lambda=1$, marked as $\Lambda_{0}$, where the Floquet operator is unitary. In the interval $0<\omega<2 \pi / 3$, there are two 2EPs within the unit circle $|\Lambda|<1$ (e.g., $\Lambda_{1}$ and $\Lambda_{2}$ in Fig. 3). These two 2EPs merge to form a $3 \operatorname{EP}\left(\Lambda_{3}\right.$ in the figure) at $\omega=2 \pi / 3$. When $\omega$ is increased further, the $3 \mathrm{EP}$ again split into two $2 \mathrm{EPs}$. One of them evolves along the positive real axis and finally arrives at $\Lambda=0$ at $\omega=\pi$. The other 2EP evolves along the negative real axis and arrives at $\Lambda_{4}$. We note that it suffices to examine the interval $0 \leqslant \omega \leqslant \pi$ due to a reflection symmetry about $\omega=\pi$ in the $\omega$ dependence of the configuration of EPs.

We provide an analytic argument to the above numerical findings. We examine the characteristic polynomial of the Floquet operator (3) given as

$$
f(z) \equiv \operatorname{det}|z-\hat{U}(\lambda)|=z^{3}+f_{2} z^{2}+f_{1} z+f_{0},
$$

where $f_{2} \equiv\left(2+\Lambda^{-1}\right) \mu, f_{1} \equiv-\left(1+2 \Lambda^{-1}\right) \mu, f_{0} \equiv-\Lambda^{-1}$ and

$$
\mu \equiv-\frac{1}{3}(1+2 \cos \omega)
$$

Note that $-1 \leqslant \mu \leqslant \frac{1}{3}$ holds for real $\omega$. Following the standard prescription to solve cubic equations [35], we introduce $p$ and $q:$

$$
p \equiv \frac{3 f_{1}-f_{2}^{2}}{9}, q \equiv \frac{2 f_{2}^{2}-9 f_{2} f_{1}+27 f_{0}}{27},
$$

from which the discriminant is defined as

$$
D \equiv-27\left(q^{2}+4 p^{3}\right) \text {. }
$$

The presence of spectral degeneracy in $\hat{U}(\lambda)$ is equivalent to the condition $D=0$. Indeed, when $D=0$ holds, the solutions of the characteristic equation $f(z)=0$ are, according to the
Cardano formula [35],

$$
z=z_{\mathrm{c}}+2\left(-\frac{q}{2}\right)^{1 / 3} \text { and } z_{\mathrm{c}}-\left(-\frac{q}{2}\right)^{1 / 3}
$$

where $z_{\mathrm{c}} \equiv-f_{2} / 3$. The latter solution is doubly degenerated. Hence, the spectrum of $\hat{U}(\lambda)$ is triply degenerate if and only if both $D=0$ and $q=0$ hold.

We show that there are, at most, four spectral degeneracy points, which are either the diabolical or exceptional point, in the $\Lambda$ plane. This is because the discriminant $D$ [Eq. (33)] is a fourth-order polynomial in $\Lambda^{-1}$ :

$$
D=\sum_{n=0}^{4} D_{n}\left(\Lambda^{-1}\right)^{4-n}
$$

with $\quad D_{0}=D_{4}=4(\mu+1) \mu^{3}, \quad D_{1}=D_{3}=4(\mu+1)(9+$ $5 \mu) \mu^{2}$, and $D_{2}=-3(\mu+1)\left(9-9 \mu-21 \mu^{2}-11 \mu^{3}\right)$.

We also show that degeneracy points form a "conjugate pair": If $\Lambda$ is a degeneracy point, then so is $\Lambda^{-1}$. The reason is that $D=0$ is a reciprocal equation, whose coefficients satisfy symmetry relations $D_{0}=D_{4}$ and $D_{1}=D_{3}$.

We now show that the triple degeneracy, obtained by solving $D=0$ and $q=0$, occurs at $(\omega, \Lambda)=(0,1)$ and $(2 \pi / 3,0)$, as shown in Fig. 3. $q$ is expressed as a polynomial of $\Lambda^{-1}$,

$$
q=\sum_{n=0}^{3} q_{n}\left(\Lambda^{-1}\right)^{3-n}
$$

with $q_{0}=2 \mu^{3} / 27, q_{1}=2 \mu^{2}(3+2 \mu) / 9, q_{2}=-\left(9-15 \mu^{2}-\right.$ $\left.8 \mu^{3}\right) / 9$, and $q_{3}=2 \mu^{2}(9+8 \mu) / 27$. $D$ and $q$ vanish simultaneously if and only if the resultant (Sylvester's determinant) $R(D, q)$ of these polynomials vanish [36]:

$$
R(D, q)=\left|\begin{array}{lccccccc}
D_{0} & D_{1} & D_{2} & D_{3} & D_{4} & 0 & 0 & 0 \\
0 & D_{0} & D_{1} & D_{2} & D_{3} & D_{4} & 0 & 0 \\
0 & 0 & D_{0} & D_{1} & D_{2} & D_{3} & D_{4} & 0 \\
0 & 0 & 0 & D_{0} & D_{1} & D_{2} & D_{3} & D_{4} \\
q_{0} & q_{1} & q_{2} & q_{3} & 0 & 0 & 0 & 0 \\
0 & q_{0} & q_{1} & q_{2} & q_{3} & 0 & 0 & 0 \\
0 & 0 & q_{0} & q_{1} & q_{2} & q_{3} & 0 & 0 \\
0 & 0 & 0 & q_{0} & q_{1} & q_{2} & q_{3} & 0 \\
0 & 0 & 0 & 0 & q_{0} & q_{1} & q_{2} & q_{3}
\end{array}\right|
$$

$$
=\frac{16}{27}(3-\mu)^{3} \mu^{9}(1+\mu)^{9} .
$$

The resultant $R(D, q)$ vanish only when $\mu$ equals either 0 or -1 . This is the condition of the triple degeneracy. These two cases (T1) and (T2) are examined in the following.

Case $(T 1) \omega=\frac{2 \pi}{3}(\mu=0)$ : As shown in Sec. III, the characteristic polynomial is $f(z)=z^{3}-\Lambda^{-1}$. Hence a 3EP locates at $\Lambda=\infty$, whose conjugate pair $\Lambda=0$ is also a 3EP.

Case (T2) $\omega=0(\mu=-1)$ : Because the characteristic polynomial is $f(z)=(z-1)^{2}\left(z-\Lambda^{-1}\right)$, the Floquet operator has eigenvalues 1 and $\Lambda^{-1}$, the former of which is doubly degenerate, and corresponding quasienergy is real. The degree of the "Hermitian" degeneracy at $\Lambda=1$, which corresponds to $\Lambda_{0}$ in Fig. 3 , is 3. Otherwise the degree is 2 .

In the following, we examine the cases (D1)-(D3) where there are, at most, doubly spectral degeneracies. 
Case (DI) $\omega=\pi\left(\mu=\frac{1}{3}\right)$ : There are three doubly degenerate points $\Lambda=\Lambda_{ \pm}$and 1 , which are the solutions of $D=0$. We have a conjugate pair of $2 \mathrm{EPs} \Lambda_{ \pm}=-(17+12 \sqrt{2})^{ \pm 1}$, which satisfy $\Lambda_{+} \Lambda_{-}=1$. Note that $\Lambda_{-}$is located inside the unit circle and corresponds to $\Lambda_{4}$ in Fig. 3. Also, there are a Hermitian degeneracy point at $\Lambda=1$.

Case (D2) $\frac{2 \pi}{3}<\omega<\pi\left(0<\mu<\frac{1}{3}\right)$ : We explain that a pair of EPs lies in the positive real axis, and the other pair lies in the negative real axis. The solution of $D=0$ of

$$
D=\frac{4 \mu^{3}(\mu+1)}{\Lambda^{2}}\left[\left(y+\frac{9+5 \mu}{2 \mu}\right)^{2}-\frac{27(1+\mu)^{2}}{4 \mu^{3}}\right]
$$

is given as

$$
y_{ \pm} \equiv \frac{1}{2 \eta^{3}}\left[-\left(3+5 \eta^{2}\right) \eta \pm\left(1+3 \eta^{2}\right)\right]
$$

with

$$
y=\Lambda+\Lambda^{-1}
$$

and

$$
\eta \equiv \sqrt{\frac{|\mu|}{3}} .
$$

Note that $0<\eta<1 / 3$ holds for $0<\mu<1 / 3$. We solve Eq. (41) to obtain $\Lambda$. Let us examine the case $y=y_{+}$first. It is straightforward to see that the solutions are

$$
\Lambda_{ \pm}^{(0)} \equiv \frac{1}{2}\left[y_{+} \pm \sqrt{\left(y_{+}\right)^{2}-4}\right]
$$

which form a conjugate pair. We show that $\Lambda_{ \pm}^{(0)}$ are positive and real. First, we examine the discriminant of the quadratic equation

$$
\left(y_{+}\right)^{2}-4=\frac{1}{4 \eta^{6}}(1-\eta)^{3}\left(1+3 \eta^{2}\right)(1-3 \eta),
$$

which is positive for $0<\eta<1 / 3$. Second, we examine the sign of $y_{+}$. We therefore have $\left|y_{+}\right|>2$ for $0<\eta<1 / 3$. Hence the sign of $y_{+}$is independent of $\eta \in(0,1 / 3)$. As $\eta \rightarrow 0+$, it is easy to see $y_{+}>0$. This implies that $y_{+}$is positive for $\eta \in(0,1 / 3)$. Hence $\Lambda_{ \pm}^{(0)}$ are also real and positive.

In a similar way, we examine the case $y=y_{-}$, where we have

$$
\Lambda_{ \pm}^{(1)} \equiv \frac{1}{2}\left[y_{-} \pm \sqrt{\left(y_{-}\right)^{2}-4}\right],
$$

which also form a conjugate pair. It is straightforward to see $\Lambda_{ \pm}^{(1)}$ are real and negative because $y_{-}<0$ and $\left(y_{-}\right)^{2}-4>0$ hold.

Case (D3) $0<\omega<\frac{2 \pi}{3}(-1<\mu<0)$ : We explain that there are two conjugate pairs of 2EPs in the $\Lambda$ plane, which can be proved in a similar way above. The difference from the above case is that these EPs are not in the real axis. As is seen in Fig. 3, the trajectories (depicted by the red curve) of two 2EPs within the unit circle correspond to this case.

We solve $D=0$ to obtain $y$ [see Eq. (41)]. The solutions are

$$
y_{c} \equiv \frac{1}{2 \eta^{3}}\left[\left(3-5 \eta^{2}\right) \eta+i\left(1-3 \eta^{2}\right)\right]
$$

and $y_{c}^{*}$, where $\eta$ is defined in Eq. (42). Note that $0<\eta<1 / \sqrt{3}$ holds for $-1<\mu<0$. For $y=y_{c}$, the solutions of Eq. (41) are

$$
\Lambda_{ \pm}^{(c)} \equiv \frac{1}{2}\left[y_{c} \pm \sqrt{\left(y_{c}\right)^{2}-4}\right]
$$

which form a conjugate pair of degeneracy points. We note that $\left[\Lambda_{ \pm}^{(c)}\right]^{*}$ are also EPs. The discriminant of the quadratic equation is

$$
\left(y_{c}\right)^{2}-4=\frac{\left[3 \eta\left(1-\eta^{2} / 3\right)+i\left(1-3 \eta^{2}\right)\right]\left(1-3 \eta^{2}\right)(3 \eta+i)}{4 \eta^{6}},
$$

which is nonzero and complex valued as long as $-1<\mu<0$. Hence $\Lambda_{ \pm}^{(c)}$ and $\left[\Lambda_{ \pm}^{(c)}\right]^{*}$ are not in the real axis.

We note that the 3EP is fragile against perturbations of $\mu$. Indeed, once $\mu$ is varied from 0 , the resultant $R(D, q)$ becomes nonzero, which implies the absence of triple degeneracy.

We proceed to examine the correspondence between the exotic quantum holonomy and the fragments of the 3EPs. To establish this, we explain the emulation of the exotic quantum holonomy with EPs by deforming the unitary cycle $C$ into non-Hermitian cycles, say, $C^{\prime}$. As for the $3 \mathrm{EP}$ case $(\omega=2 \pi / 3)$, we refer the reader to the previous section and Fig. 2. Because there is only a single EP, the resultant Riemann surface structure is rather simple.

Due to the rupture of 3EP into several EPs, the Riemann surface structure becomes complicated. We here examine the case $\omega=\pi / 6$, where the eigenspace anholonomy is equivalent to the case $\omega=2 \pi / 3$ (see Fig. 1). There are two 2EPs, $\Lambda_{1}$ and $\Lambda_{2}$ (Fig. 3).

In addition to the configuration of these EPs, we need to take into account the branch cuts of quasienergies in the $\Lambda$ plane to discuss the deformation of the adiabatic cycle. Here we repeat
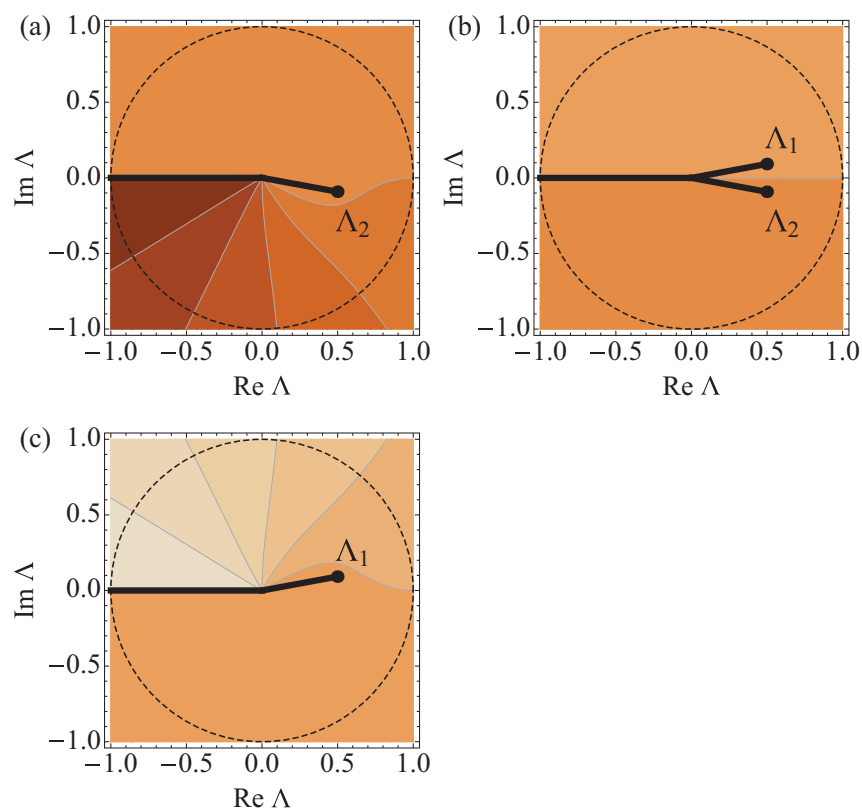

FIG. 4. (Color online) Contour plots of $\operatorname{Re} E_{M}$ in the $\Lambda$ plane with (a) $M=-1$, (b) $M=0$, and (c) $M=1$. We choose $\omega=\pi / 6$, where a 3EP is split into two 2EPs indicated by filled circles. Other parameters are the same as in Fig. 2. 


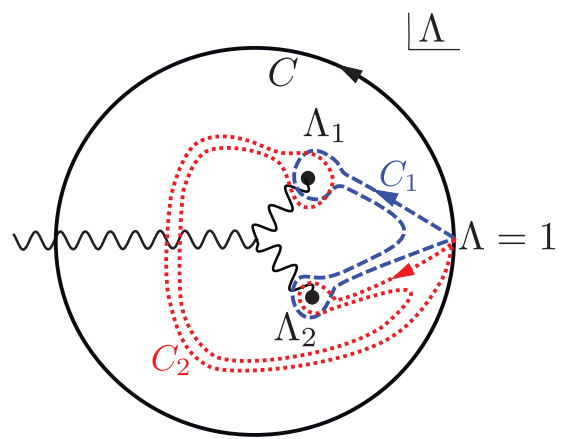

FIG. 5. (Color online) A schematic explanation of two nonHermitian cycles $C_{1}$ (dashed lines) and $C_{2}$ (dotted lines) that emulate a unitary cycle $C$ in the $\Lambda$ plane for the quantum kicked spin $J=1$ and $\omega=\pi / 6$ (see Fig. 4). $C$ start from $\Lambda=1$ and encircle EPs $\Lambda_{1}$ and $\Lambda_{2}$.

the same procedure to carry out the analytic continuation (see Sec. IV) to obtain the Riemann sheets of quasienergies, as shown in Fig. 4. As a result, we find how the Riemann sheets of quasienergies are connected by the EPs and the branch cuts. $E_{-1}$ and $E_{0}$ degenerate at $\Lambda_{2}$. On the other hand, $E_{0}$ and $E_{1}$ degenerate at $\Lambda_{1}$.

There are two kinds of non-Hermitian cycles that emulate the exotic quantum holonomy induced by the unitary cycle $C$. Typical examples $C_{1}$ and $C_{2}$ are shown in Fig. 5. They are obtained through smooth deformations of $C$. Along $C_{1}$, we first encircle $\Lambda_{1}$, where $E_{M=0}$ and $E_{M=-1}$ are interchanged, and then we encircle $\Lambda_{2}$, where $E_{M=-1}$ and $E_{M=0}$ are interchanged. The composition of these permutation results in the cyclic permutation given by Eq. (29).

On the other hand, we need to take into account the presence of branch cuts for the analysis of the cycle $C_{2}$. Along $C_{2}$, we first encircle $\Lambda_{2}$, where $E_{M=-1}$ and $E_{M=0}$. Then, we need to come across a branch cut. Because of this, $E_{M}$ becomes $E_{M-1}$. Next we encircle $\Lambda_{2}$, and then come across the branch cut again. Thus we conclude that the adiabatic cycle along $C_{2}$ also induces the cyclic permutation shown in Eq. (29).

We summarize the analysis of the $J=1$ case to clarify the correspondence between the exotic quantum holonomy and the bifurcation of EPs. While the exotic quantum holonomy along the adiabatic cycle $C$ is kept intact for $0<\omega \leqslant \pi$, the configuration of EPs is sensitive to $\omega$. There is a single $3 \mathrm{EP}$ within the unit circle of the $\Lambda$ plane at $\omega=2 \pi / 3$. It is straightforward to obtain the non-Hermitian cycle, which encircle the 3EP (e.g., $C^{\prime}$ in Fig. 2), to emulate the exotic quantum holonomy. As for the two 2EPs case $(\omega \neq 2 \pi / 3)$, two cycles that enclose the 2EPs are combined to emulate the exotic quantum holonomy. The contributions from the two 2EPs are not interchangeable. They need to be combined in exact order to correctly emulate the exotic quantum holonomy (see $C_{1}$ and $C_{2}$ in Fig. 5).
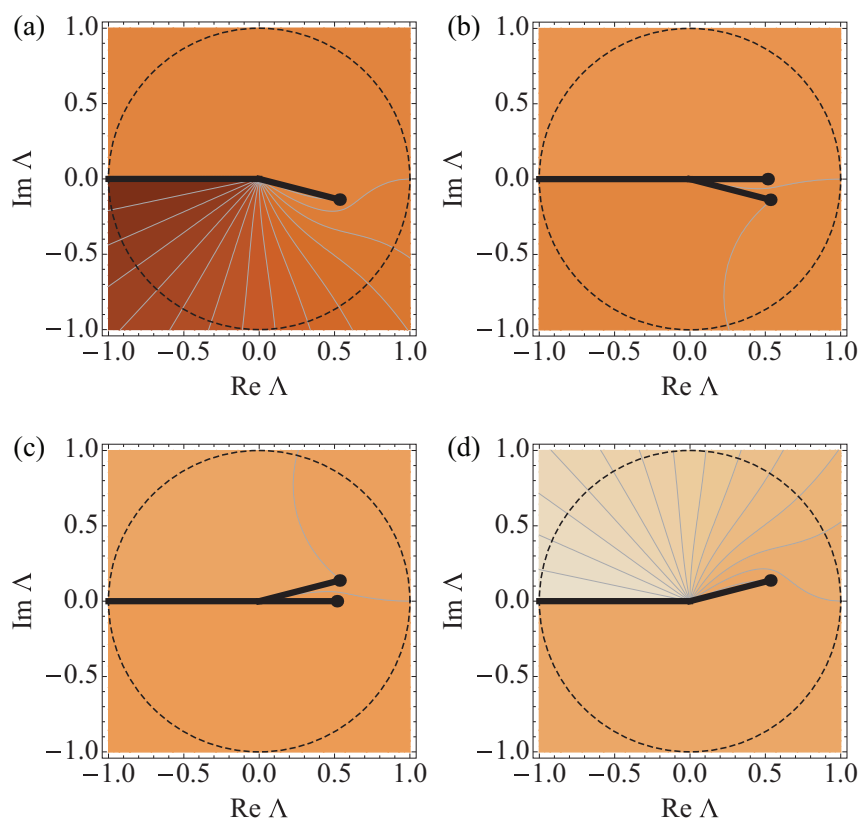

FIG. 6. (Color online) Contour plots of $\operatorname{Re} E_{M}$ in the $\Lambda$ plane with (a) $M=-3 / 2$, (b) $M=-1 / 2$, (c) $M=1 / 2$, and (d) $M=3 / 2$. Other parameters are $J=3 / 2$ and $\omega=\pi / 8$. There are three 2EPs, which merge at $\omega=\pi / 2$ (not shown here) to compose a 4EP. Other conventions are the same as in Fig. 2.

A similar analysis can be carried out for the quantum kicked top with an arbitrary $J$. We depict the $J=3 / 2$ case in the vicinity of a 4EP in Fig. 6.

\section{SUMMARY}

We have examined the exotic quantum holonomy in a family of quantum kicked tops $J$, against an adiabatic cycle $C$, where the strength of the kick is increased. This model exhibits the exotic quantum holonomy for an arbitrary value of the parameter of the unperturbed top $\omega$, except at the resonant points. This model has a $(2 J+1)$-EP for a specific value of $\omega$. It is shown that an infinitesimal perturbation can split $(2 J+1)$-EP into $2 J$ 2EPs with $J=1$ and $3 / 2$. Analytic argument is provided for $J=1$. We have observed an intricate interplay of the exotic quantum holonomy and EPs for both the $(2 J+1)$-EP case and the 2EPs case.

\section{ACKNOWLEDGMENTS}

This research was supported by NRF funded by the Ministry of Science, ICT and Future Planning (Grant No. 2013R1A1A2011438), and by the Japan Ministry of Education, Culture, Sports, Science and Technology under Grant No. 24540412.
[1] T. Kato, J. Phys. Soc. Jpn. 5, 435 (1950).

[2] M. V. Berry, Proc. Roy. Soc. London A 392, 45 (1984).

[3] F. Wilczek and A. Zee, Phys. Rev. Lett. 52, 2111 (1984).
[4] T. Cheon, Phys. Lett. A 248, 285 (1998).

[5] A. Tanaka and M. Miyamoto, Phys. Rev. Lett. 98, 160407 (2007). 
[6] M. Miyamoto and A. Tanaka, Phys. Rev. A 76, 042115 (2007).

[7] T. Cheon and A. Tanaka, Europhys. Lett. 85, 20001 (2009).

[8] T. Cheon, A. Tanaka, and S. W. Kim, Phys. Lett. A 374, 144 (2009).

[9] A. Tanaka and T. Cheon, Phys. Rev. A 82, 022104 (2010).

[10] A. Tanaka, S. W. Kim, and T. Cheon, Europhys. Lett. 96, 10005 (2011).

[11] N. Yonezawa, A. Tanaka, and T. Cheon, Phys. Rev. A 87, 062113 (2013).

[12] E. Farhi, J. Goldstone, S. Gutmann, and M. Sipser, arXiv:quant$\mathrm{ph} / 0001106$.

[13] A. Tanaka and K. Nemoto, Phys. Rev. A 81, 022320 (2010).

[14] M. Berry, N. Balazs, M. Tabor, and A. Voros, Ann. Phys. (NY) 122, 26 (1979).

[15] Ya. B. Zel'dovich, Sov. Phys. JETP 24, 1006 (1967).

[16] M. Sadgrove, M. Horikoshi, T. Sekimura, and K. Nakagawa, Phys. Rev. Lett. 99, 043002 (2007).

[17] J. Chabé, G. Lemarié, B. Grémaud, D. Delande, P. Szriftgiser, and J. C. Garreau, Phys. Rev. Lett. 101, 255702 (2008).

[18] S. Chaudhury, A. Smith, B. E. Anderson, S. Chose, and P. Jessen, Nature (London) 461, 768 (2009).

[19] A. Morello et al., Nature (London) 467, 687 (2010).

[20] T. Kato, Perturbation Theory for Linear Operators (SpringerVerlag, Berlin, 1980), Chap. II.

[21] R. Uzdin, A. Mailybaev, and N. Moiseyev, J. Phys. A: Math. Theor. 44, 435302 (2011).
[22] M. V. Berry and R. Uzdin, J. Phys. A: Math. Theor. 44, 435303 (2011).

[23] R. Uzdin and N. Moiseyev, Phys. Rev. A 85, 031804 (2012).

[24] S. W. Kim, T. Cheon, and A. Tanaka, Phys. Lett. A 374, 1958 (2010).

[25] J.-W. Ryu, S.-Y. Lee, and S. W. Kim, Phys. Rev. A 85, 042101 (2012).

[26] S.-Y. Lee, J.-W. Ryu, S. W. Kim, and Y. Chung, Phys. Rev. A 85, 064103 (2012).

[27] A. Tanaka, N. Yonezawa, and T. Cheon, J. Phys. A: Math. Theor. 46, 315302 (2013).

[28] W. D. Heiss, J. Phys. A. 41, 244010 (2008).

[29] E. M. Graefe, U. Günther, H. J. Korsch, and A. E. Niederle, J. Phys. A. 41, 255206 (2008).

[30] M. Combescure, J. Stat. Phys. 59, 679 (1990).

[31] S. Ghose, R. Stock, P. Jessen, R. Lal, and A. Silberfarb, Phys. Rev. A 78, 042318 (2008).

[32] M. Srinivas and E. Davies, Opt. Acta 28, 981 (1981).

[33] M. Mohseni, P. Rebentrost, S. Lloyd, and A. Aspuru-Guzik, J. Chem. Phys. 129, 174106 (2008).

[34] See, e.g., N. Moiseyev, Non-Hermitian Quantum Mechanics (Cambridge University Press, New York, 2011).

[35] NIST Handbook of Mathematical Functions, edited by F. W. J. Olver, D. W. Lozier, R. F. Boisvert, and C. W. Clark (Cambridge University Press, Cambridge, 2010), Chap. 1.

[36] B. van der Waerdern, Moderne Algebra (Frederick Ungar, New York, 1949), Vol. I, Chap. 4. 